\title{
Paradigm Shift in Reliability Monitoring
}

\author{
M. Perry, P. Schroeder, D. Vuncannon, R. Dipper, T. Bull \\ Nortel Northern Telecom) \\ RTP, NC 27709-3478, USA, mperry@nortel.com
}

\author{
A. Nilsson \\ North Carolina State University \\ Dept. of ECE, Raleigh, NC 27695, USA, nilsson@ncsu.edu \\ B. Peters \\ Ameritech \\ 220 N. Meridian, Room 910, Indianapolis, IN 46204, USA
}

\begin{abstract}
Reliability and maintenance of telecommunications equipment is evolving and continues to evolve. Improved hardware, development of software engineering principles, and better understanding of procedures have reduced system downtime. This is reflected in more stringent downtime specifications in the telecommunications industry. The makeup of failures leading to downtime has also changed. Advances in digital equipment have dramatically reduced hardware problems. Although software has also improved, more is demanded of it, and it has not improved at the same rate as hardware. Procedural techniques have also improved--better user interfaces, and improvements in process have led to fewer failures. However, maintenance personnel maintain more equipment than before, and in consequence, procedural failure rates have not improved as fast as those for hardware. Software and procedural problems--not hardware--are now the dominant reasons for outages. Not only do they cause most outages, but the public perceives these outages to be worse. Yet the current in-service auditing of telecommunications equipment may still be based on a paradigm of preventing outages caused by relatively unreliable hardware. Auditing--the inspection and testing of communications equipment--is performed on a regular basis. The purpose of auditing telecommunications switching equipment is to improve system reliability. For duplex equipment (equipment consisting of two identical units, one of which takes over if the other breaks down) auditing takes place on both the active and inactive units. If problems are found, repairs can be made before service is impacted. Despite this clear benefit, audits also incur costs. Auditing invokes software which itself has a failure rate. Audit testing can lead to two types of incorrect conclusion, both of which can lead to unnecessary repairs, or to a dangerous misperception that the equipment is working properly. The first type of incorrect conclusion is a false positive--the audit shows there is a problem
\end{abstract}

Performance of Information and Communication Systems U. Körner \& A. Nilsson (Eds.)

C) 1998 IFIP. Published by Chapman \& Hall 
when none exists. The second is a false negative--the audit shows there is no problem when one exists. Reducing the incorrect audit conclusions will reduce the dominant failures of today--software and procedural. Since audits incur both costs and benefits, the natural conclusion is that audits should be run neither too frequently nor too infrequently. Yet there is a lack of guidelines on how often to perform audits. Our hypothesis is that audits are run too frequently for today's mix of failures. We develop a detailed reliability model of a generic duplex module for switching equipment. Our intent is to provide a useful methodology and some results that are independent of any manufacturer's equipment. The model shows that selecting the correct audit frequency saves significantly on downtime and maintenance costs. The results of this modeling were then applied to live equipment in the field. The reported results are very encouraging--81\% less outages, $79 \%$ less software traps, and 39\% fewer hardware failure reports.

\section{Keywords}

Reliability, audits, switching equipment, outages, maintenance, duplex.

\section{INTRODUCTION}

The reliability of switching equipment is changing. Section 12 , of the LATA Switching Systems Generic Requirements (LSSGR),[1] states that:

"A group of 24 or more trunks should be out-of-service for no more than 20 minutes per year"

A more recent supplement to this document[2], specifies downtime affecting 100 or more trunks states:

"Digital Trunk Multitermination Downtime shall be no more than 1.2 minutes per year"

Improved reliability and more stringent requirements result from improved hardware, software, redundancy, and telephone company procedures. Hardware reliability in particular has improved dramatically. Most personal computers users will agree that modern microprocessors and memory are far more reliable than they were 10 years ago. The same is true of switching equipment. More rigorous testing, structured and object-oriented programming, improved operating systems, and memory protection have all helped reduce software failure rates. Better understanding of user-friendly interfaces and human factors have also led to improved procedural failure rates.

However these reductions do not fully characterize the change in reliability; the failure mix has changed as well. Although hardware is now extremely reliable and rarely fails, and software has improved, more is demanded of software, so it now accounts for a greater proportion of errors. Procedures also have improved, but larger, more complicated systems are being maintained by fewer people, so there are proportionately more procedural failures. Furthermore, the public perceives outages caused by procedural or software failures to be worse. Therefore if significant gains in reliability are to be made, software and procedural failure rates must be targeted. 
To further reduce software failure rates, the types of the software failure must be understood. For this paper, the software failures we consider are those that are sufficiently severe to bring down a unit of duplex switching equipment.

At the risk of over-simplification, software failures fall into two categories: maintenance and call processing. Call processing software errors cause failures less often than maintenance errors because call processing code is more thoroughly tested through use of simulated traffic and in general is more visible[3]. Also, there are fewer exceptions in call processing software paths than in maintenance software. Maintenance software, being less visible with more exceptions, is less well tested. Our focus for software will be on reducing maintenance software errors.

There are many approaches to reducing procedural errors. Some major examples follow.

- Improve processes: Use two repair persons instead of one, even though this will increase costs.

- Make equipment easier to repair: Create better documentation and improve human factors design.

- Increase necessary maintenance actions: Ensure true failures are not ignored.

- Reduce unnecessary maintenance actions: Maintenance actions are inherently risky since repairs are intrusive and increase the chances of other failures. For instance, the wrong card might be replaced when a circuit pack is being changed.

Our focus for procedural problems will be on increasing necessary maintenance actions and reducing unnecessary maintenance actions.

Fortunately, both maintenance software failures and procedural problems can be addressed by a single operational decision to modify the continuous auditing of hardware.

Operational tests or audits form a large part of maintenance software. Used for testing switch equipment and taking corrective action, audits are scheduled by the unit's operating system, and run as maintenance processes which check the status of the communications equipment. In duplex configurations, both units are monitored and tested. When problems are found they are reported and corrective action is taken. Audits for checking hardware sanity are run frequently. For example, many hardware components in the trunk peripherals for Nortel's DMS100 switching equipment are checked every 10 to 400 seconds.

Audits bring the benefit of being able to make repairs before service is affected. If the inactive unit of a duplex peripheral has a detectable fault on a circuit pack, the audit will find it, report it to the operations personnel, and a repair will be made. Without the audit, the fault could remain undiscovered, so that when an error occurred on the sound unit, the backup (inactive) unit would be unable to assume duty; resulting in an outage.

Audits come at a price, however. Since they are tasks scheduled by the operating system, audits can "trap" (suffer a software failure); they can also report a problem where none exists (false positive); and they can also fail to report a 
problem when one exists (false negative). Software traps and false positive reports have similar results: the unit is taken out of service for repair. Unnecessary failures and repairs increase maintenance costs and decrease reliability. Furthermore, when the false positive rate is high, telephone personnel begin to mistrust the alarms, and may not respond to real problems. False negative reports decrease the benefit of testing, since audits need to find problems to provide value. They also lead to a faulty system state--which increases the chances of software failure.

The conclusion, therefore, is that audits should be run neither too frequently nor too infrequently.

It seems likely that the frequency of auditing has not kept pace with the changing failure rates. Formerly, under the paradigm that hardware was "unreliable", frequent hardware checks made sense--even at the expense of more procedural and maintenance software failures. But under the current paradigm that hardware is very reliable it would not make sense to risk the increase in software and procedural failure rates associated with frequent auditing.

In summary, the hypothesis is that running the audits less frequently will improve reliability and reduce maintenance costs. As a result, there will be fewer software failures in maintenance code, fewer procedural outages, and greater responsiveness to real problems because there will be fewer false positives.

The costs and benefits of auditing were weighed in a detailed analytic and simulation modeling exercise. This exercise not only provided a means of determining how much auditing should take place, but also gave further insight on whether audits were scheduled too frequently. Best estimates were then made for more appropriate audit schedules. These new schedules were tested in the field. The results, while not definitive, are encouraging.

\section{MODELS}

The model for determining the correct audit schedule needed to be relatively complex.

To understand the state of a duplex peripheral, the state of both active and inactive units must be known. This state depends on reality, perception, and failure-type.

Reality refers to whether the unit can perform service. Perception refers to whether the unit believes itself to be capable of performing service. Many states have a mismatch between the reality and perception which can cause inappropriately risky actions or lack of appropriate actions. If a unit is down, for example, but appears to be up, actions (such as repair, or switching activity to the inactive unit) will not take place. Conversely, if a unit appears to be down when it is up, expensive, risky, and unnecessary repairs will be performed. This mismatch between perception and reality is driven by the interaction between failures and audits.

There are three types of failure. The first, Type 1, includes failures that are immediately obvious to the system. These cause events in the peripheral which are understood and reported by the software. For example, if a facility interface 
card fails, frame synchronization is lost, the unit reporting the event runs tests to isolate the failure, and issues a report.

The second type of failure includes those where the system depends on the audit system to find them. The failure will not trigger operational events that would be understood by the system, since some errors are only visible when specific diagnostics are run. (Diagnostics are run periodically to check sanity in the units.) These are shown as Type 2 failures in the state table later in this document.

The third type of failure includes those the system does not find. They do not trigger events recognized by the software, nor are they detectable by the periodic audits, since diagnostic coverage is not 100 percent. Such failures are serviceaffecting, so complaining customers or observant telephone company personnel will eventually discover them. These are shown as Type 3 failures in the state table below.

\subsection{State Descriptions}

Not all states generated by all combinations of the variables need be described because most states are not feasible. For instance, if both units are up and are perceived as being up, it makes no sense to categorize the failures since there are none (Not Applicable, NA, in the last two columns in Table 1). Similarly, when a unit is perceived as being down, it is unnecessary to describe the failure type, since the failure or perception of failure is already known, and is unimportant because a repair action will inevitably occur.

Table 1 contains the list of system states. State 1 is the typical healthy state--both units are up and perceived as up. State 5 is an outage--when both units are down and perceived as being down. States 7 and 8 are "unnecessary" outages where the active unit is down, but perceived as up, and the inactive unit is able to assume service if directed to do so.

Table 1: System states

\begin{tabular}{|c|c|l|c|c|c|c|}
\hline State & $\begin{array}{l}\text { Perception } \\
\text { Active Unit }\end{array}$ & $\begin{array}{l}\text { Reality } \\
\text { Active Unit }\end{array}$ & $\begin{array}{l}\text { Perception } \\
\text { Inactive } \\
\text { Unit }\end{array}$ & $\begin{array}{l}\text { Reality } \\
\text { Inactive Unit }\end{array}$ & $\begin{array}{l}\text { Type 2 or } \\
\text { Type 3 } \\
\text { Active }\end{array}$ & $\begin{array}{l}\text { Type 2 or } \\
\text { Type 3 } \\
\text { InActive }\end{array}$ \\
\hline 1 & up & up & up & up & NA & NA \\
\hline 2 & up & up & down & NA & NA & NA \\
\hline 3 & up & up & up & down & NA & Type 2 \\
\hline 4 & up & up & up & down & NA & Type 3 \\
\hline 5 & down & NA & down & NA & NA & NA \\
\hline 6 & up & down & down & NA & Type 2 & NA \\
\hline 7 & up & down & down & NA & Type 3 & NA \\
\hline 8 & up & down & up & up & Type 2 & NA \\
\hline 9 & up & down & up & up & Type 3 & NA \\
\hline 10 & up & down & up & down & Type 2 & Type 2 \\
\hline 11 & up & down & up & down & Type 3 & Type 2 \\
\hline 12 & up & down & up & down & Type 2 & Type 3 \\
\hline 13 & up & down & up & down & Type 3 & Type 3 \\
\hline
\end{tabular}


Using Markov modeling [4] [5] and numerical methods [6], the probabilities of being in state $i--\pi_{i}(i=1, \ldots, 13)$ can be determined. The outage states are $5,6, \ldots 13$. The sum of these probabilities, multiplied by the number of minutes in a year, gives the expected number of downtime minutes per year--D.

$$
\mathrm{D}=60 * 24 * 365.25 * \sum_{\mathrm{i}=5}^{13} \pi_{\mathrm{i}}
$$

In this section the objective is to determine audit frequencies that result in nearoptimal reliability as measured by downtime and incidents. The analysis was done by independently considering the performance of individual hardware components in a duplex standby (inactive) peripheral. The failure rate, audit rate, false negative rate, false positive rate, and Type 3 failure discovery rate were estimated for the auditable hardware components. The best way to calculate the failure rates was to examine a large population of field data, software-generated LOGs, return rates, and fault-found rates on a per-circuit pack basis. Although this calculation was made for Nortel products in the US market, the results presented in this paper are based on failure rates in a generic duplex switching peripheral.

For a unit with 20 hardware components, using downtime reliability requirements as specified in [1], industry estimates on repair and dispatch times as in [1,2], breakdown of failures as in [1,2], and a procedure as in [7], failure rates ranging between 500 and 4000 FITs (failures in a billion hours) were found to be reasonable.

There were, however, certain limitations to the analytic modeling and engineering approach. The time between audits was closer to being fixed than to the classical exponential assumption. Also, a real-life unit consists of many hardware components with failure and audit rates of their own. Ideally, the analytical model should be composed of all the individual hardware components and their own failure and audit rates, but such a model would be too big to interpret and draw practical conclusions. Instead, the model used an aggregate of many hardware components and related failure and audit parameters. The method used to engineer the audit frequencies was to use the analytic model on individual hardware components one by one using relevant parameters. A simulation accounting for the fixed audit times and multiple hardware components is described in Section 4.0. It was used to understand the impact of these issues.

Other important parameters included false negative probability, Type 3 failure discovery time on the active unit, Type 3 failure discovery time on the inactive unit, mean time to repair, and probability of destructive repair $\left(p_{d}\right)$. These parameters and their assumed values are discussed in Section 3.3, Assumptions.

The engineering methodology included determining the FIT rate, the probability of false positive, and the apparency level (this is the fraction of failures that are Type 1 failures) on a per hardware component basis. The time between audits on the inactive unit was varied discretely between 10 and 2880 seconds, and the time between audits on the active unit was varied discretely from 10 to 2880 seconds 
for each fixed time between audits on the inactive unit. The FIT rate, false positive probability, and apparency level were then varied individually to yield other sets of results. The FIT rate was varied discretely from 500 to 4000 . (As previously discussed, this should cover a wide range of FIT rates for both the hardware components and the generic peripheral.) The false positive probability took on two values, $10^{\wedge}-6$ and $10^{\wedge}-7$.The apparency level (see section 3.1) took on values of $0.0,0.5$, and 1.0 for the active unit, with an apparency level of 0.0 assumed for the inactive unit in all cases. As a result, for each ordered triple of FIT rate, false positive probability, and apparency level, "good" audit frequencies were obtained for both the active and inactive units. A summary of the results is given below, together with an example of how to use the Table.

\subsection{Audit Frequency Engineering Table}

The following table is indexed on hardware component FIT rate, false positive probability, and apparency level. An apparency level of 0.5 (Appar=0.5) means that $50 \%$ of the failures are of Type 1 and the other $50 \%$ is the total of Type 2 and Type 3. The false-negative probability is used to partition the Type 2 and Type 3 failures. For each ordered triple of these parameters, effective times between audits on both the active and inactive units are found in Table 2. The analytical results assume the capability of having distinct audit frequencies for both the active and inactive units.

\section{Table 2 Audit Engineering Table for Time Between Audits}

\begin{tabular}{|c|c|c|c|c|c|c|}
\hline TS & $\begin{array}{l}\text { Active } \\
\text { Appar=0 } \\
P_{\mathrm{fp}}=10^{-6}\end{array}$ & $\begin{array}{l}\text { Active } \\
\text { Appar }=0 \\
P_{f p}=10^{-7}\end{array}$ & $\begin{array}{l}\text { Active } \\
\text { Appar }=0.5 \\
P_{\text {fp }}=10^{-6}\end{array}$ & $\begin{array}{l}\text { Active } \\
\text { Appar }=0.5 \\
P_{\mathrm{fp}}=10^{-7}\end{array}$ & $\begin{array}{l}\text { Active } \\
\text { Appar }=0.5 \\
P_{\text {fp }}=10^{-6}\end{array}$ & $\begin{array}{l}\text { Active } \\
\text { Appar }=0.5 \\
\mathrm{P}_{\mathrm{fp}}=10^{-7}\end{array}$ \\
\hline 500 & & & & 12 & & \\
\hline 000 & & $(180$ & )) & $(18$ & & \\
\hline 000 & 80) & $(90$, & $(360$ & $(180$, & 12 & $\longdiv { 1 2 8 }$ \\
\hline 000 & 80) & $(90$, & $(240$ & $(120$, & & $(288$ \\
\hline 000 & $(180,2880)$ & $(90,2880)$ & $(240,2880)$ & $(120$ & & $(288$ \\
\hline
\end{tabular}

\subsection{Using the Engineering Table}

The Engineering Table gives an effective audit frequency for a given peripheral hardware component. To use Table 2, the FIT rate, the probability of a false positive diagnostic, and the apparency level of failures for the hardware component must be known. For example, if a hardware component is known to have a FIT rate of approximately 1000 FITs, a diagnostic false positive probability of $10^{\wedge}-6$, and an apparency level of approximately 0.5 , the Table shows the "best" time between audits to be 360 seconds on the active unit and 2880 seconds on the inactive unit.

An inter-audit time of 2880 seconds may not be truly "optimal", but rather the upper bound on the inter-audit time for the numerical results. Extending the audit times further may bring additional benefits, but of diminishing returns. 


\subsection{Parameters and Assumptions}

Other parameters used in Table 2 are defined in the following list, which also includes assumed values for each parameter.

False Negative Probability: This was assumed to be 0.1 ; and after discussions with maintenance personnel in central offices, and research into industry specifications, diagnostic coverage of 90 percent was assumed.

Type 3 failure discovery times for the active and inactive units were assumed to be 0.25 and 2.0 hours respectively. These figures were arrived at after discussions with telephone personnel regarding various products in their networks.

Mean Time to Repair was assumed to be one hour [1].

Probability of Destructive Repair: The probability that a procedural error will cause additional problems on an active unit while a problem is being investigated was assumed to be 0.01 . This assumption was corroborated in conversations with workers at two major telephone operating companies.

These assumed parameter values were "conservative"; that is, they favor more frequent auditing. For example, 90 percent coverage is quite high. If lower coverage were assumed, the optimal audit frequency would be less.

\subsection{Engineering Graphs}

Some examples of how reliability responds to the model parameters are shown in the graphs that follow. Table 2 was developed from graphs like these.

Outages per Year per Peripheral

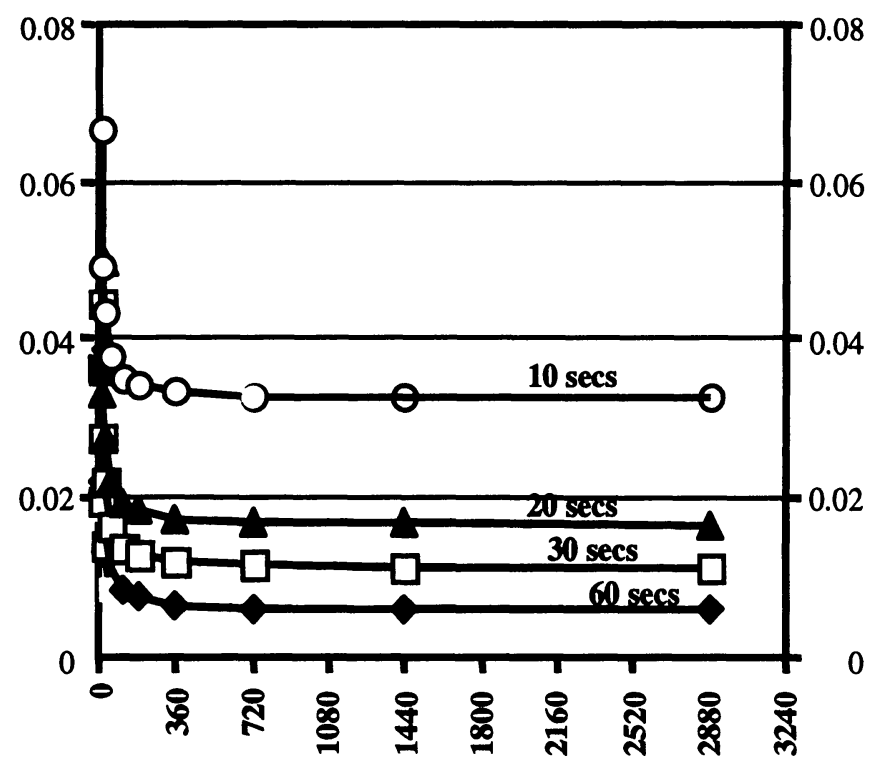

Active Inter Audit Time (seconds)

FITs $=500$, Incidents, Active Unit failures not apparent, False positive $=10^{\wedge}-6$

Outages per year per peripheral with inactive inter audit time as a parameter. 
Downtime Hours per Year per Peripheral

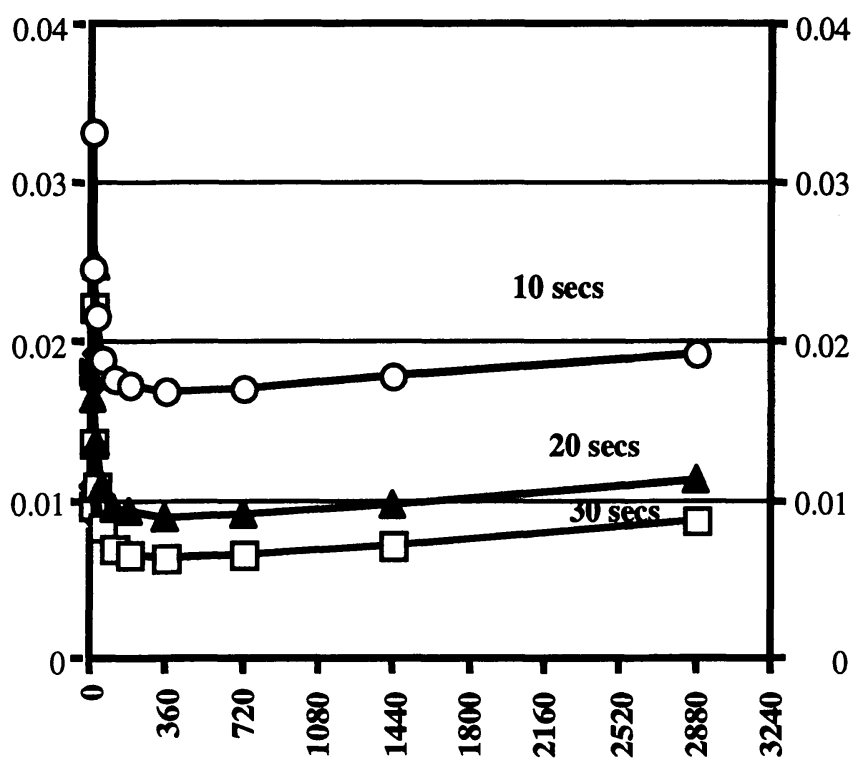

Active Inter Audit Time (seconds)

FITs=500, Downtime (hours), Active Unit failures not apparent, False positive $=10^{\wedge}-6$

Hours of downtime per year per peripheral with inactive inter audit time as a parameter.

\subsection{Modeling Conclusions}

Given that hardware reliability has improved vastly over the last 10 years, it is easy to schedule audits too frequently. The engineering approach allows more appropriate audit frequencies to be selected in a scientific manner.

\section{$4 \quad$ SIMULATION MODELING}

A simulation model was developed for a duplex peripheral consisting of a number of hardware components, each with its own family of parameters including failure rates, audit rate, and repair rates as described in Section 3.0. The simulation could execute with either exponential or deterministic times between audits. Only a limited number of scenarios could be covered because there were so many parameters.

When the simulated system had a single hardware component and exponential service times, the simulation results and analytical results matched, as was to be expected. Moreover, when the simulated system had multiple hardware components and deterministic times between audits, the results for optimal audit rates were similar to those for the analytic model. Optimizing the audit rate for the individual components with exponential times seemed to match well with the best audit rates for the full system with multiple hardware components in the 
simulation model. Nor did the determinist times impact optimum audit frequency (See [7] for a sensitivity of the fixed testing times as compared with the exponential.) This indicated that engineering the audit frequency as described in section 3.0 was appropriate. Results depended heavily on input data that had been carefully estimated through analyzing industry data and specifications. A field experiment was undertaken to further verify the approach and hypothesis.

\section{EXPERIMENTAL VALIDATION}

An audit frequency experiment was conducted to validate (or invalidate) the analytic and simulation modeling results described previously. Actual field results were used to lend credence to the hypothesis that auditing is being carried out too frequently. An experiment was undertaken to reduce the audit frequency by a factor of 10 , using a simple procedure that was easily applied to 13 peripherals in a large, feature-rich DMS switch in an urban area on the Ameritech network.

A special monitoring system installed in the office with the reduced audit frequencies collected details on the office performance. These details, which included system state tracking, audit reports, software failures, and hardware failures, were downloaded, aggregated, and reported on a daily basis.

To run a complete experiment it was necessary to estimate how much data should be collected. The data was measured in terms of peripheral in-service years, taking into account both the number of peripherals in the experiment and the length of time being monitored. For example, 10 peripherals monitored for one half-year would provide five years of in-service data. Applying a method for determining sample size for comparative experiments in [8] showed that it would take 100 in-service years to be 80 percent confident of detecting a 20 percent difference in outages between systems with the old and new audit frequencies. Less data would be needed to understand the differences in software errors and false positives, since these events are less rare.

So far 12 in-service years of data for the experimental group has been collected, results to date are promising. Eleven similar-sized switches with hardware and software similar to those of the experimental switches were also monitored over the same period of time. The measures were averaged and compared with the experimental group with the reduced audits. The results are summarized in Table 3.

Table 3 Experimental Results Percent Reduction in the Experimental Group

\begin{tabular}{|c|c|}
\hline Measure & Percent Reduction \\
\hline Outages & $81 \%$ \\
\hline Software Traps & $73 \%$ \\
\hline Hardware Failure & $39 \%$ \\
\hline
\end{tabular}

All measures are favorable. Not only was the experimental switch more reliable, it was also cheaper to maintain, since fewer maintenance actions were required.

It should be noted that slowing the audits does not affect the number of true hardware problems that are eventually found, the cost is that some failures are discovered later. However, the number of false positive hardware reports 
decreases, and the data supports this. Software traps are also interesting; the data shows that running audits less frequently is helpful.

On the qualitative level, the site engineer monitoring the switch said

"We have not seen any problems with the reduced audit frequency. It has not contributed to any peripheral outages or any other problem."

Another operating company that has changed audit frequency in a test switch made similar qualitative observations, however no special monitoring was done. Plans are now in place to change the audit frequencies in four new switches.

Although there is insufficient data to make statistical statements at traditional levels of confidence, the data on running audits less frequently is very promising.

\section{SUMMARY}

Changes in reliability and failures in switching equipment have been discussed. Hardware reliability has improved dramatically. Software and procedures have also improved also, but not as much as hardware. Analysis focused on maintenance software, since more problems occur there than in call processing software. For procedural problems, effort focused on minimizing unnecessary maintenance actions. Continuous auditing of the system was then considered, and the hypothesis made that the amount of auditing currently taking place was based on the outdated premise that hardware was unreliable and needed very frequent checking. We speculated that current auditing schedules did not adequately account for the improved hardware reliability. An analytic model was developed that accounted for both the costs and the benefits of auditing. This approach was partially validated in simulation, and then used to engineer the audit frequencies. A field experiment was conducted on a feature-rich DMS switch where the audit frequency on 13 peripherals had been reduced by a factor of 10 . The results of that experiment, although not definitive, are promising.

7 REFERENCES

[1] Bellcore, TR-TSY-000512, LSSGR: Reliability, Section 12, Issue 3, February 1990.

[2] Bellcore, TR-TSY-000512, LSSGR: Reliability, Section 12, Issue 3, February 1990; Supplement 1, August 1993.

[3] Houck, D.J., K.S. Meier-Hellstern, F. Saheban, and R.A. Skoog, Failure and Congestion Propagation Through Signaling Controls, ITC-14, Antibes Juan-lesPins France, June 1994.

[4] Kleinrock, L., Queuing Systems Volume I: Theory, New York, 1975.

[5] Bellcore, SR-TSY-001171, Methods and Procedures for System Reliability Analysis, Issue 1, May 1989.

[6] Stark, P., Introduction to Numerical Methods, New York Macmillan Publishing Co., Inc., 1970.

[7] Perry, M., D. Hickman and A. Nilsson, Optimal Execution Tests and System Reliability, Globecom, London, November 1996.

[8] Snedecor G. and Cocharan G, Statistical Methods, Iowa State University Press, August 1989. 